\title{
RELACIÓN ENTRE LOS FACTORES SOCIOECONÓMICOS, SOCIODEMOGRÁFICO Y LA PUBLICIDAD BTL EN LA INCIDENCIA DE LA OBESIDAD INFANTIL EN CUENCA- ECUADOR
}

\author{
RELATIONSHIP BETWEEN THE SOCIOECONOMIC, SOCIODEMOGRAPHIC AND BTL \\ PUBLICITY FACTORS IN THE INCIDENCE OF CHILDHOOD OBESITY IN CUENCA-ECUADOR
}

\author{
Marco Antonio Ríos Ponce \\ Facultad Ciencias de la Administración \\ Universidad del Azuay \\ Cuenca-Azuay-Ecuador \\ mrios@uazuay.edu.ec
}

\section{Diana Carolina Arízaga Toledo \\ Facultad Ciencias de la Administración Universidad del Azuay Cuenca-Azuay-Ecuador dianacaroarizagat@yahoo.com}

\author{
Juan Francisco Álvarez Valencia \\ Facultad Ciencias de la Administración \\ Universidad del Azuay \\ Cuenca-Azuay-Ecuador \\ falvarez@uazuay.edu.ec
}

Joffre Sebastián Arteaga Huiracocha

Facultad Ciencias de la Administración

Universidad del Azuay

Cuenca-Azuay-Ecuador

arteagasebastian32@gmail.com

Fecha de recepción: 03/05/2018 - Fecha de aprobación: 30/08/2018

\section{RESUMEN}

El objetivo del presente estudio es conocer si existe una correlación entre los factores socioeconómicos, sociodemográficos y la publicidad Below the Line (BTL) en la incidencia de la obesidad infantil, la metodología utilizada fue una investigación exploratoria-descriptiva, utilizando herramientas cualitativas, que ayudaron a recopilar información por medio de grupos focales, entrevistas a expertos y entrevistas a profundidad lo cual dio paso a la realización de la investigación cuantitativa por medio de encuestas a padres de familia con niños con sobrepeso u obesidad infantil. Como resultado de esta investigación, encontramos que el uso de dispositivos electrónicos genera el impacto, no por la publicidad BTL, sino por el uso excesivo de estos dispositivos provocando sedentarismo y la deficiente comunicación por parte del ente gubernamental de las políticas para reducir la obesidad infantil.

PALABRAS CLAVE: Marketing Social; Publicidad; Below the Line; Obesidad Infantil; Comunicación.

\section{ABSTRACT}

An exploratory-descriptive research was developed to determine the influence that BTL, advertising and electronic media had on children and their parents when choosing food

\footnotetext{
"Visión de Futuro" Año 16, Volumen No 23 N¹, Enero - Junio 2019 - Pág. 157 - 176

URL de la Revista: http://revistacientifica.fce.unam.edu.ar/

URL del Documento: http://revistacientifica.fce.unam.edu.ar/index.php?option=com content\&view=article\&id=492\&ltemid=101

ISSN 1668 - 8708 - Versión en Línea

E-mail: revistacientifica@fce.unam.edu.ar
} 
products. Two research methods were applied. A qualitative research gathered information through focus group, interviews with experts and interviews. Then, a quantitative research was carried out through surveys applied to parents of overweight children. Finally, the dato was proessed and the socio-economic and socio-demographic results were obtained. This information allowed to identify the eating behaviors of the families in Cuenca-Ecuador.

KEY WORDS: Social Marketing; Advertising; Below the Line; Childhood Obesity; Communication.

\section{INTRODUCCIÓN}

Investigar, escribir y hablar sobre marketing puede resultar más complicado de lo que parece, debido a que tiene gran trayectoria a través de la historia, porque el marketing existió desde casi el inicio de la humanidad. El origen de la palabra Marketing, proviene de Estados Unidos, a pesar de que no se conoce con exactitud quién fue el creador de esta palabra y tampoco el año en el que se comenzó a utilizar, existen distintas especulaciones sobre su origen, pero se conoce que este término hacía referencia a estudiar y aplicar distintas estrategias para obtener una efectiva comercialización de bienes y servicios en un mercado específico. El marketing ha ido evolucionando gracias a la creación de distintos medios de comunicación como son: imprenta, radio y televisión (ATL), pero esto no ha sido suficiente, por lo que, en la actualidad, las estrategias de marketing se llegan a perfeccionar con las estrategias de BTL (Below The Line) y el avance de la tecnología, donde ya no se enfoca en el producto sino en el valor de las personas.

El Marketing es una forma de organizar un conjunto de acciones y procesos a la hora de crear un producto para crear, comunicar y entregar valor a los clientes y para manejar las relaciones; su finalidad es beneficiar a la organización satisfaciendo a los clientes (American Marekting Association, 2013).

La obesidad infantil es un tema controversial que se ha venido tratado en todo el mundo, por lo que La Organización Mundial de la salud - OMS - (Organización Mundial de la Salud, 2016), define "El sobrepeso y la obesidad como un acumulación anormal o excesiva de grasa que supone un riesgo para la salud". Según datos de la Encuesta Nacional de Salud y Nutrición (Freire \& Ramírez), en Ecuador se registra que entre la edad de 5 a 11 años, el índice de obesidad se triplica llegando al 29,9\%.

El objetivo de la investigación es identificar si existe correlación entre la publicidad BTL y la obesidad infantil en la ciudad de Cuenca, las razones para que exista esta enfermedad en la ciudad, los estímulos de las decisiones de compra en los representantes de niños entre

\footnotetext{
“Visión de Futuro" Año 16, Volumen N² 23 Nº1, Enero - Junio 2019 - Pág. 157 - 176

URL de la Revista: http://revistacientifica.fce.unam.edu.ar/

URL del Documento: http://revistacientifica.fce.unam.edu.ar/index.php?option=com content\&view=article\&id=492\&ltemid=101 
un rango de edad de 5 a 11 años y variables de consumo, por lo que se realizará una investigación cualitativa y cuantitativa para conocer del tema y poder sacar resultados coherentes sobre la obesidad.

\section{DESARROLLO}

\section{Metodología}

Se realizó una investigación exploratoria - descriptiva, la cual consistió en conocer los comportamientos predominantes de los consumidores a través de la descripción de sus actividades y proceso de decisión. Se aplicaron dos tipos de investigación: cualitativa y cuantitativa.

\section{Fase 1: Investigación cualitativa}

Se utilizaron técnicas de recolección de datos como: entrevistas a tres diferentes tipos de expertos, para este estudio se consideró a expertos en marketing, en nutrición y pediatras, para conocer su opinión sobre los factores nutricionales y publicitarios que inciden en la obesidad infantil; se elaboró un grupo focal de 12 padres de familia y representantes de la edad entre 27 a 40 años de edad que tienen niños de 5 a 11 años, para conocer la alimentación, la influencia que tiene la publicidad BTL y las formas de entretenimiento que tienen sus niños; entrevistas a profundidad a cuatro padres de familia de las mismas características de los participantes en el grupo focal, donde se trató sobre la alimentación que tienen los niños en sus hogares y la posible influencia de la publicidad BTL en los infantes, los pasatiempos de los niños, los programas y políticas gubernamentales para erradicar la obesidad infantil. Toda la investigación cualitativa recaudó muchos temas importantes para llegar a conocer donde radica el problema de la obesidad infantil y poder continuar con la investigación cuantitativa, para realizar el informe de esta fase 1 se apoyó en el software ATLAS TI.

\section{Fase 2: Investigación cuantitativa}

Se realizó un muestreo por conveniencia, tomando 300 hogares de la ciudad de Cuenca Urbana como referencia de la obesidad infantil para que la investigación sea significativa. Cabe recalcar que el muestreo por conveniencia es un muestreo no probabilístico, "la elección de los elementos no depende de la probabilidad sino de las causas relacionadas con las características de la investigación o de quién hace la muestra" (Hernández Sampieri \& Fernández Collado, 2006, pág. 241), donde las personas son seleccionadas dependiendo de la accesibilidad y disponibilidad de los investigadores.

\footnotetext{
“Visión de Futuro" Año 16, Volumen N² 23 Nº1, Enero - Junio 2019 - Pág. 157 - 176

URL de la Revista: http://revistacientifica.fce.unam.edu.ar/

URL del Documento: http://revistacientifica.fce.unam.edu.ar/index.php?option=com content\&view=article\&id=492\&/temid=101 
Se enviaron 4.500 encuestas a familias con niños entre 5 a 11 años de edad de la ciudad de Cuenca por medio de escuelas fiscales, privadas y fiscomisionales; al no ser obligatorio el llenado de esta encuesta, regresaron 2.500 contestadas, de las cuales 300 encuestas fueron efectivas para la investigación. Para considerar efectiva tenía que responder afirmativamente en la pregunta filtro si tenía algún grado de sobrepeso u obesidad infantil.

El procedimiento y análisis estadístico de datos se realizó con ayuda del programa IBM SPSS Statistics versión 23 y los resultados se presentaron en tablas dinámicas para su mejor comprensión.

\section{Desarrollo del contenido}

La accesibilidad que tienen las personas a la información de todo tipo hace que sus hábitos alimenticios se modifiquen constantemente, debido a que la publicidad BTL está lanzando nuevos productos contantemente y convenciendo a las personas que cada vez son mejores, por lo que cambia radicalmente el estilo de vida de las familias y se hace parte de una cultura el ingerir alimentos procesados que van directo a la mesa. Es impresionante todo el conocimiento que tienen las personas sobre los distintos tipos de alimentos que se ofrecen en el mercado, sin embargo, a pesar de que conocen cuáles son los alimentos buenos y malos, se observa que todas las personas dentro y fuera de su hogar tienen una dieta muy variada de alimentos que son a la vez nutritivos con proteínas y otros alimentos altos en grasa, combinándolos constantemente sin tener buenos resultados para un futuro.

La alimentación de las personas va muy arraigada con el nivel socioeconómico y la educación que tienen; la primera, es que mientras el ingreso económico por familia sea más bajo, su alimentación es menos nutritiva y balanceada. Actualmente vemos publicidad en todos lados, muy llamativa, sobre alimentos como snacks, comida rápida, jugos azucarados, gaseosas, etc. que termina convenciendo desde los padres hasta los más chicos para el consumo inmediato y por consiguiente vienen los cambios alimenticios; porque si los alimentos comprados llegan a gustarle al niño/a, los padres lo comprarán frecuentemente.

Estos cambios alimenticios que tiene la gente, vienen dados por varios factores que poco a poco hacen que la gente consuma productos que son perjudiciales para su salud. Uno de los factores principales es el sedentarismo, este tema es de gran preocupación ya que viene dado por el exceso en el uso de la computadora y el celular, pero eso no es todo. Muchas empresas de alimentos buscan generar ventas constantemente, por lo tanto, desarrollan cualquier tipo de campaña publicitaria para ofrecer un producto que es elaborado con componentes que llevan los requerimientos mínimos para poder ser comercializados dentro del país. Al elaborar estrategias para una comunicación efectiva en medios electrónicos como redes sociales, páginas web, aplicaciones y también la publicidad en las calles de la ciudad

\footnotetext{
“Visión de Futuro" Año 16, Volumen N² 23 Nº1, Enero - Junio 2019 - Pág. 157 - 176 
ofreciendo un producto que es dañino para la salud de las personas, no se está realizando una responsabilidad social, sino que la empresa se enfoca netamente en el beneficio económico.

Otro factor sumamente importante es el tiempo, tanto padres como madres de familia tienen trabajos a tiempo completo, lo que los obliga a comprar productos que sean sencillos de cocinar en un menor tiempo, o también, compran alimentos ya hechos, únicamente para el consumo inmediato. Según los padres de familia, son las bebidas gaseosas el principal producto que causa sobrepeso y obesidad infantil, debido a que son los productos con mayor accesibilidad a todas las personas, se pueden encontrar en cualquier lugar y además lo prefieren para refrescarse. Además de las bebidas azucaradas también existe un gran consumo de dulces por parte de los niños, los cuales también tienen un alto contenido de azucares procesados y son adquiridos especialmente por su forma, presentación y precio, que son atractivos tanto para padres como para niños.

Finalmente, se observa que los esfuerzos del Gobierno no han sido del todo eficaces a pesar de que se han implementado nuevas normas a las cuales las empresas han tenido que adaptarse para vender sus productos en Ecuador, por lo que las empresas deben tener una mayor responsabilidad social enfocada en la salud de las personas, sobre todo de los niños. Estas campañas de marketing social pueden ayudar a las personas que no tienen mucha información sobre temas de nutrición y concientizarlos para que así puedan cambiar sus hábitos alimenticios, que afectan su salud a futuro.

El Gobierno ha generado cierto control sobre los alimentos y productos que se venden dentro de los establecimientos educativos, en este caso, el control de los bares que han expandido su menú con productos más nutritivos y al mismo tiempo eliminando otros productos que son realmente perjudiciales para la salud de un niño, que son altos en grasa.

La idea de la semaforización de los productos es una buena iniciativa para que la gente pueda tener más cuidado en la selección de los productos, sin embargo, existen falencias en este concepto, ya que la gente puede llegar a priorizar ciertos productos con componentes que no ayudan al crecimiento del niño/a sustituyendo por otros productos que si complementan a la vida del niño/a con una dieta balanceada y nutritiva.

\section{Investigación Cualitativa}

Se muestran los resultados de mayor relevancia de las distintas intervenciones a expertos en marketing, nutrición y pediatría con respecto a la temática de estudio, por medio de un análisis en el Software ATLAS.ti.

\footnotetext{
“Visión de Futuro" Año 16, Volumen N² 23 Nº1, Enero - Junio 2019 - Pág. 157 - 176 


\section{obesidad Marketing Disminuir ético Infantil Control parental}

\section{Gráfico No1. Nube ATLAS.ti Expertos en Marketing}

Fuente: Elaboración Propia

Los expertos en marketing aseguran:

- El marketing está para generar valor y satisfacción.

- Las diferentes estrategias de marketing deben responder a un código ético.

- Los representantes deben poner límites en consumo de ciertos productos.

- Existe una corresponsabilidad entre los padres y las empresas por responder adecuadamente a las necesidades de los niños.

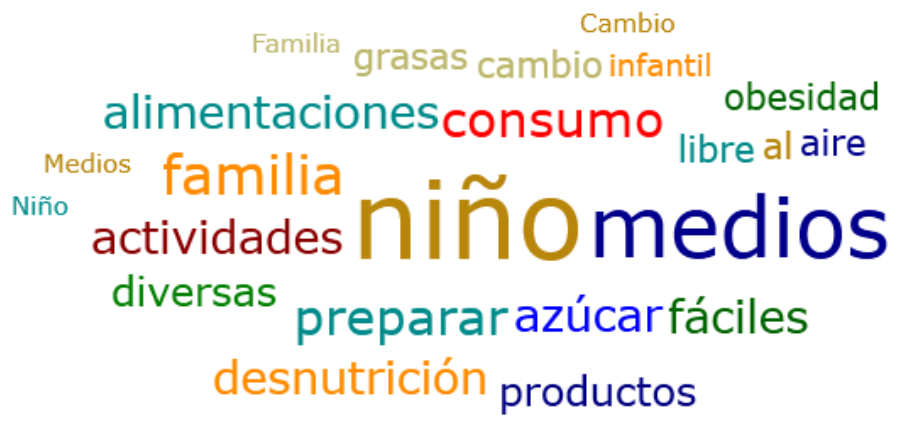

Gráfico N2. Nube ATLAS.ti Expertos en Nutrición

Fuente: Elaboración Propia

Los nutricionistas analizan e indican que:

- La globalización provoca cambios en los hábitos de consumo de las personas.

- Los niños pasan cada vez menos tiempo en actividades al aire libre.

- Incremento en productos con altos contenidos hipercalóricos y de fácil acceso para los niños.

- Los hábitos que su núcleo familiar tenga afectarán directamente en su salud física y mental.

\footnotetext{
"Visión de Futuro" Año 16, Volumen N² 23 N¹, Enero - Junio 2019 - Pág. 157 - 176

URL de la Revista: http://revistacientifica.fce.unam.edu.ar/

URL del Documento: http://revistacientifica.fce.unam.edu. ar/index.php?option=com content\&view=article\&id=492\&/temid=101

ISSN 1668 - 8708 - Versión en Línea

E-mail: revistacientifica@fce.unam.edu.ar
} 


\section{control compensación \\ infantil $\underset{\text { mujer }}{\text { variada }}$ cambio $_{\text {rol }}$ padres \\ leche Alimentación ${ }_{\text {obesidad }}$ \\ familiarmaterna Falta ${ }_{\text {Hábitos }}$ niños familiainstitución calórico}

\section{Gráfico N3. Nube ATLAS.ti Expertos en Pediatría}

Fuente: Elaboración Propia

Los pediatras exponen que:

-Disminuye el control a los niños con la inserción de la mujer en el ámbito laboral.

- Los padres no deben compensar a sus hijos con alimentos hipocalóricos.

- Las familias se alimentan según sus conocimientos y posibilidades económicas.

- Los hábitos familiares repercuten en las preferencias alimenticias del niño.

- Se debe establecer rutinas al igual que límites sobre lo que pueden o no comer sus hijos.

\section{Investigación de campo}

Tablas de frecuencia: 
Tabla No1 Estado Civil del representante

\begin{tabular}{|l|c|c|}
\hline \multicolumn{3}{|c|}{ ESTADO CIVIL DEL REPRESENTANTE } \\
\hline & Frecuencia & Porcentaje válido \\
\hline Soltero/a & 31 & 10,5 \\
\hline Casado/a & 197 & 65,2 \\
\hline Divorciado/a & 41 & 13,9 \\
\hline Unión Libre & 25 & 8,4 \\
\hline Viudo/a & 6 & 2 \\
\hline Total & 300 & 100 \\
\hline
\end{tabular}

Fuente: Arízaga y Arteaga (2018)

\section{ESTADO CIVIL DEL} REPRESENTANTE

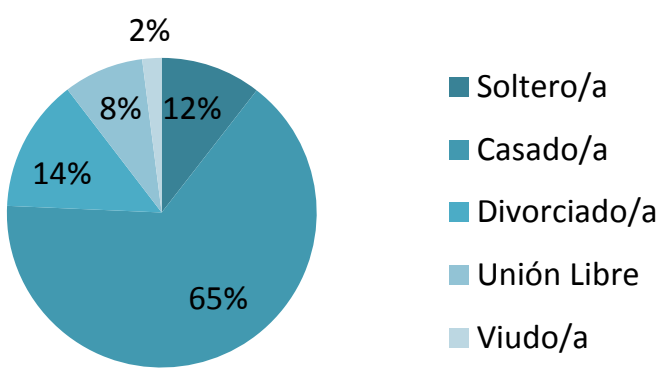

Figura No1. Estado Civil del Representante Fuente: Arízaga y Arteaga (2018)

Tabla N2. Nivel de Instrucción del representante

\section{NIVEL DE INSTRUCCIÓN DEL REPRESENTANTE}

\begin{tabular}{|c|c|c|}
\hline & Frecuencia & Porcentaje válido \\
\hline Básico & 35 & 11,9 \\
\hline Secundario & 84 & 28,6 \\
\hline Tercer Nivel & 144 & 46,9 \\
\hline Cuarto Nivel & 37 & 12,6 \\
\hline Total & 300 & 100 \\
\hline
\end{tabular}

Fuente: Arízaga y Arteaga (2018)

\section{NIVEL DE INSTRUCCIÓN DEL REPRESENTANTE}

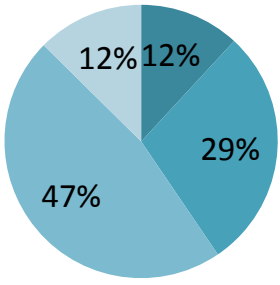

- Básico

- Secundario

Tercer Nivel

Cuarto Nivel

Figura N2. Nivel de instrucción del representante

Fuente: Arízaga y Arteaga (2018)

Tabla N3. Edad del Niño/a

\begin{tabular}{|c|c|c|}
\hline & EDAD DEL NIÑO/A \\
\hline & Frecuencia & Porcentaje válido \\
\hline 5 años & 21 & 7,0 \\
\hline 6 años & 34 & 11,3 \\
\hline 7 años & 36 & 12,0 \\
\hline 8 años & 37 & 12,3 \\
\hline 9 años & 56 & 18,7 \\
\hline 10 años & 60 & 20,0 \\
\hline 11 años & 56 & 18,7 \\
\hline
\end{tabular}

Fuente: Arízaga y Arteaga (2018)

\section{EDAD DEL NIÑO/A}

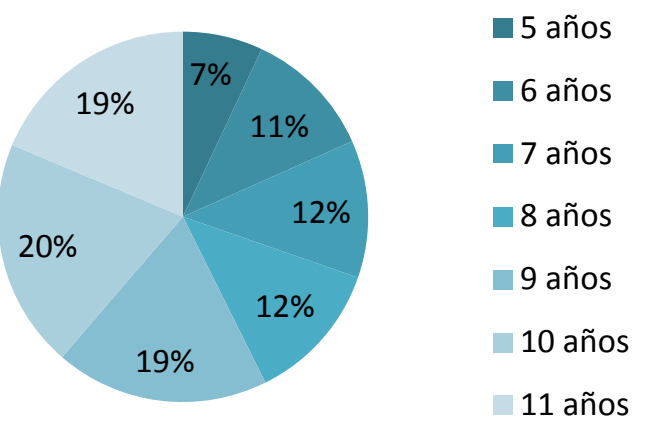

Figura N3. Edad del niño/a

Fuente: Arízaga y Arteaga (2018)

"Visión de Futuro" Año 16, Volumen Nº 23 N¹, Enero - Junio 2019 - Pág. 157 - 176

URL de la Revista: http://revistacientifica.fce.unam.edu.ar/

URL del Documento: http://revistacientifica.fce.unam.edu.ar/index.php?option=com content\&view=article\&id=492\&ltemid=101

ISSN 1668 - 8708 - Versión en Línea

E-mail: revistacientifica@fce.unam.edu.ar 
Tabla N4. Hogares con Internet

\begin{tabular}{|c|c|c|}
\hline \multicolumn{3}{|c|}{ HOGARES CON INTERNET } \\
\hline & Frecuencia & $\begin{array}{c}\text { Porcentaje } \\
\text { válido }\end{array}$ \\
\hline $\mathrm{Si}$ & 244 & 80,9 \\
\hline No & 56 & 19,1 \\
\hline Total & 300 & 100 \\
\hline
\end{tabular}

Fuente: Arízaga y Arteaga (2018)

\section{HOGARES CON INTERNET}

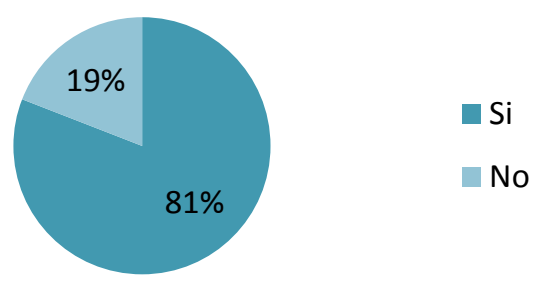

Figura $N^{0} 4$. Existe servicio de internet en el hogar

Fuente: Arízaga y Arteaga (2018)

Tabla N5. Hogares con Juegos electrónicos

\section{HOGARES CON JUEGOS ELECTRÓNICOS}

\begin{tabular}{|c|c|c|}
\hline & Frecuencia & $\begin{array}{c}\text { Porcentaje } \\
\text { válido }\end{array}$ \\
\hline $\mathrm{Si}$ & 111 & 38,5 \\
\hline $\mathrm{No}$ & 189 & 61,5 \\
\hline Total & 300 & 100 \\
\hline
\end{tabular}

Fuente: Arízaga y Arteaga (2018)

\section{HOGARES CON JUEGOS ELECTRÓNICOS}

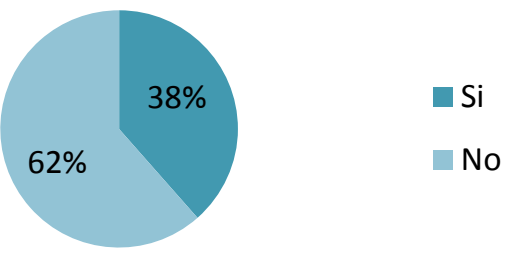

Tabla N6. Ingresos Totales del Hogar INGRESOS TOTALES DEL HOGAR

\begin{tabular}{|c|c|c|}
\hline & Frecuencia & $\begin{array}{c}\text { Porcentaje } \\
\text { válido }\end{array}$ \\
\hline Menor a $\$ 500$ & 56 & 19,4 \\
\hline$\$ 500$ a $\$ 800$ & 78 & 22,9 \\
\hline$\$ 801$ a $\$ 1100$ & 51 & 17,7 \\
\hline$\$ 1101$ a $\$ 1400$ & 30 & 10,4 \\
\hline$\$ 1401$ a $\$ 1700$ & 24 & 8,3 \\
\hline$\$ 1701$ a $\$ 2000$ & 24 & 8,3 \\
\hline Mayor a $\$ 2000$ & 37 & 12,8 \\
\hline Total & 300 & 100 \\
\hline
\end{tabular}

Fuente: Arízaga y Arteaga (2018)

INGRESOS TOTALES DEL HOGAR

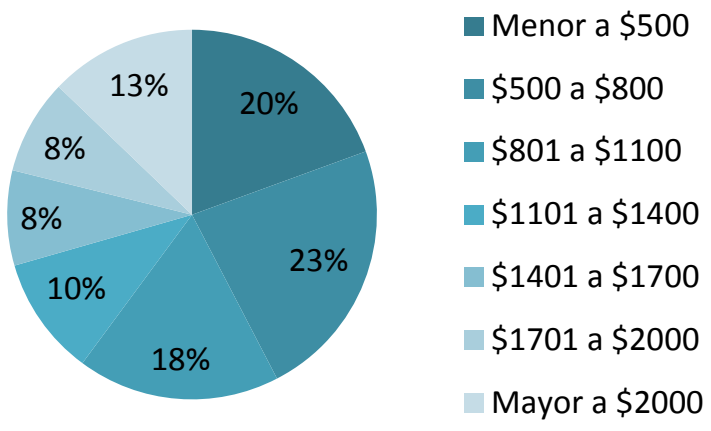

Figura N6. Ingresos totales del hogar Fuente: Arízaga y Arteaga (2018)

Tabla N7. Diagnóstico médico sobre el niño

\begin{tabular}{|c|c|c|}
\hline \multicolumn{3}{|c|}{$\begin{array}{l}\text { EN LA ÚLTIMA VISITA AL MÉDICO SU HIJO } \\
\text { PRESENTÓ: }\end{array}$} \\
\hline & Frecuencia & $\begin{array}{l}\text { Porcentaje } \\
\text { válido }\end{array}$ \\
\hline $\begin{array}{l}\text { Algún grado de } \\
\text { sobrepeso }\end{array}$ & 265 & 88,3 \\
\hline $\begin{array}{l}\text { Algún grado de } \\
\text { obesidad }\end{array}$ & 35 & 11,7 \\
\hline Total & 300 & 100 \\
\hline
\end{tabular}

Figura N5. Hogares con Juegos Electrónicos Fuente: Arízaga y Arteaga (2018)

\footnotetext{
"Visión de Futuro" Año 16, Volumen N²3 N¹, Enero - Junio 2019 - Pág. 157 - 176

URL de la Revista: http://revistacientifica.fce.unam.edu.ar/

URL del Documento: http://revistacientifica.fce.unam.edu.ar/index.php?option=com_content\&view=article\&id=492\&ltemid=101 
EN LA ÚLTIMA VISITA AL MÉDICO

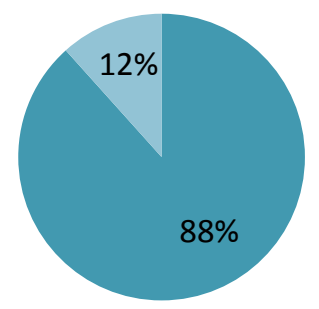

Algún grado de sobrepeso

Algún grado de obesidad

Figura N7. Diagnóstico de médico sobre el niño Fuente: Arízaga y Arteaga (2018)

Tabla N08. Accesibilidad del niño/a a medios electrónicos

\begin{tabular}{|c|c|c|}
\hline \multicolumn{3}{|c|}{ ¿EL NIÑO TIENE MEDIOS ELECTRÓNICOS? } \\
\hline & Frecuencia & $\begin{array}{c}\text { Porcentaje } \\
\text { válido }\end{array}$ \\
\hline $\mathrm{Si}$ & 110 & 37,9 \\
\hline No & 190 & 62,1 \\
\hline Total & 300 & 100 \\
\hline
\end{tabular}

Fuente: Arízaga y Arteaga (2018)

\section{¿EL NIÑO TIENE MEDIOS ELECTRÓNICOS?}

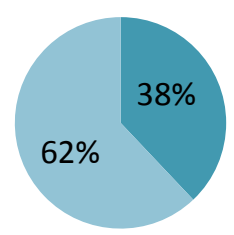

Figura N88. Accesibilidad del niño/a a medios electrónicos

Fuente: Arízaga y Arteaga (2018)
Tabla N9. Horas de entretenimiento electrónico del niño/a por día

\begin{tabular}{|c|c|c|}
\hline \multicolumn{3}{|c|}{$\begin{array}{l}\text { EXPOSICIÓN DEL NIÑO A LA PUBLICIDAD EN } \\
\text { MEDIOS ELECTRÓNICOS }\end{array}$} \\
\hline & Frecuencia & $\begin{array}{l}\text { Porcentaje } \\
\text { válido }\end{array}$ \\
\hline Redes Sociales & 58 & 23,1 \\
\hline $\begin{array}{c}\text { Juegos y } \\
\text { aplicaciones }\end{array}$ & 175 & 50,2 \\
\hline Páginas Web & 47 & 18,7 \\
\hline $\begin{array}{l}\text { Consolas de Video } \\
\text { Juego }\end{array}$ & 20 & 8 \\
\hline Total & 300 & 100 \\
\hline
\end{tabular}

\section{EXPOSICIÓN DEL NIÑO/A A LA PUBLICIDAD EN MEDIOS ELECTRÓNICOS}

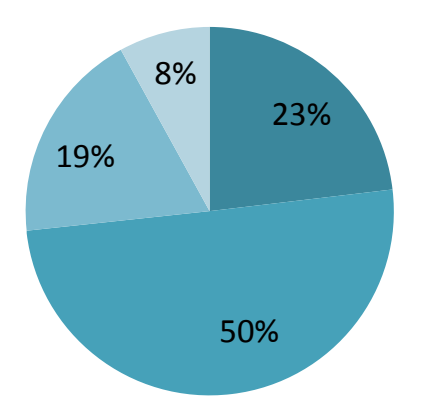

$$
\begin{aligned}
& \text { Redes Sociales } \\
& \text { Juegos y } \\
& \text { aplicaciones } \\
& \text { Páginas Web } \\
& \text { Consolas de } \\
& \text { Video Juego }
\end{aligned}
$$

Figura N9. Horas de entretenimiento electrónico del niño/a por día Fuente: Arízaga y Arteaga (2018) 
Tabla $N^{\circ} 10$. Mayor impacto de la publicidad para el niño/a

\begin{tabular}{|c|c|c|}
\hline \multicolumn{3}{|c|}{ MAYOR IMPACTO DE LA PUBLICIDAD PARA EL } \\
NIÑO & Frecuencia & $\begin{array}{c}\text { Porcentaje } \\
\text { válido }\end{array}$ \\
\hline Transporte Público & 39 & 14,1 \\
\hline Vallas Publicitarias & 146 & 44,2 \\
\hline Carritos de Comida & 115 & 41,7 \\
\hline Total & 300 & 100 \\
\hline
\end{tabular}

Fuente: Arízaga y Arteaga (2018)

MAYOR IMPACO DE LA PUBLICIDAD PARA EL NIÑO

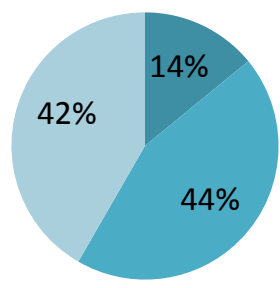

$$
\begin{aligned}
& \text { Transporte } \\
& \text { Público } \\
& \text { Vallas } \\
& \text { Publicitarias } \\
& \text { Carritos de } \\
& \text { Comida }
\end{aligned}
$$

Figura $\mathbf{N}^{\circ}$ 10. Mayor impacto de la publicidad para el niño/a

Fuente: Arízaga y Arteaga (2018)

Tabla N011. Invasión de la tecnología en los espacios verdes para los niños/as

\begin{tabular}{|c|c|c|}
\hline \multicolumn{3}{|c|}{ INVACIÓN DE LA TECNOLOGÍA EN ESPACIOS } \\
VERDES \\
\hline & Frecuencia & Porcentaje válido \\
\hline Si & 258 & 85,3 \\
\hline No & 42 & 14,7 \\
\hline Total & 300 & 100 \\
\hline
\end{tabular}

Fuente: Arízaga y Arteaga (2018)

\section{INVACIÓN DE LA TECNOLOGÍA EN ESPACIOS VERDES}

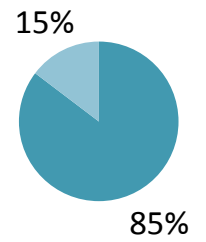

Figura $\mathrm{N}^{0} 11$. Invasión de la tecnología en los espacios verdes para los niños/as Fuente: Arízaga y Arteaga (2018)

Tabla $\mathbf{N}^{0} 12$. Horas de Entretenimiento electrónico del niño/a al día

\begin{tabular}{|c|c|c|}
\hline \multicolumn{3}{|c|}{ HORAS DE ENTRETENIMIENTO ELECTRÓNICO } \\
DEL NIÑO AL DÍA \\
\hline & Frecuencia & $\begin{array}{c}\text { Porcentaje } \\
\text { válido }\end{array}$ \\
\hline Ninguno & 37 & 12,6 \\
\hline Menos de 2 horas & 208 & 68,7 \\
\hline 2-5 horas & 48 & 16,3 \\
\hline 5-8 horas & 6 & 2 \\
\hline Más de 8 horas & 1 & 0,3 \\
\hline Total & 300 & 100 \\
\hline
\end{tabular}

Fuente: Arízaga y Arteaga (2018)

\section{HORAS DE ENTRETENIMIENTO} ELECTRÓNICO DEL NIÑO/A AL DÍA

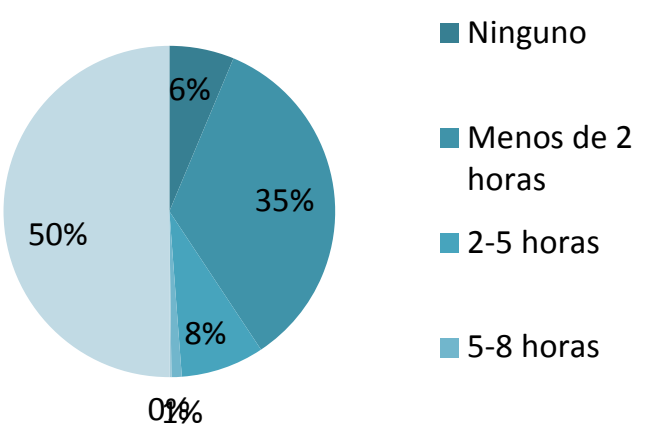

Figura $\mathbf{N}^{0} 12$. Horas de Entretenimiento electrónico del niño/a al día

Fuente: Arízaga y Arteaga (2018)

\footnotetext{
“Visión de Futuro" Año 16, Volumen No 23 N¹, Enero - Junio 2019 - Pág. 157 - 176

URL de la Revista: http://revistacientifica.fce.unam.edu.ar/

URL del Documento: http://revistacientifica.fce.unam.edu.ar/index.php?option=com content\&view=article\&id=492\&/temid=101 


\section{Conclusiones de las tablas de frecuencia}

Es necesario tomar en cuenta los principales factores que causan la obesidad infantil, identificando en primera instancia el conocimiento de los padres de familia y representantes en el tema, quienes generalmente son los encargados de guiar la alimentación y la nutrición de los niños. Cada adulto o delegado es el responsable de la cantidad de alimentos sanos o alimentos dañinos que el niño debe ingerir. Por lo que se pudo determinar, en base a la investigación por entrevistas, que muchos de los padres de familia no están bien informados o capacitados sobre los distintos tipos de alimentos que los niños necesitan diariamente. Por esa razón, un actor fundamental para controlar de una mejor manera la obesidad infantil, es el gobierno. Es necesario establecer políticas y reglamentos dentro y fuera de las escuelas. A pesar de que ya se han concretado y puesto en marcha algunas de ellas, podemos observar un incremento de esta enfermedad en los niños, lo que quiere decir que no hay un seguimiento controlado tanto del gobierno como de los padres de familia o representantes.

Es importante que los actores principales, puedan controlar la publicidad y el uso excesivo de los medios electrónicos que traen consigo todo tipo de publicidad y entre ellos, la mala alimentación con imágenes y videos de comida altos en grasas y calorías.

\section{Tablas dinámicas}

Tabla N¹3. Niños/as que tienen juegos electrónicos en su hogar con respecto a las horas que dedican al entretenimiento

\begin{tabular}{|c|c|c|c|c|c|c|c|c|c|c|c|c|c|}
\hline & \multicolumn{12}{|c|}{$\begin{array}{c}\text { ELULAR, TV, COMPUTAI } \\
\text { JUEGOS)? }\end{array}$} \\
\hline & & \multicolumn{2}{|c|}{ Ninguno } & \multicolumn{2}{|c|}{$\begin{array}{c}\text { Menos de } 2 \\
\text { horas }\end{array}$} & \multicolumn{2}{|c|}{ 2-5 horas } & \multicolumn{2}{|c|}{ 5-8 horas } & \multicolumn{2}{|c|}{$\begin{array}{c}\text { Más de } 8 \\
\text { horas }\end{array}$} & \multicolumn{2}{|c|}{ Total } \\
\hline & & 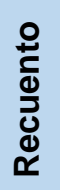 & $\begin{array}{c}\% \text { del } \\
\text { N de } \\
\text { fila }\end{array}$ & 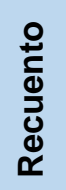 & $\begin{array}{c}\% \text { del } \\
\text { N de } \\
\text { fila }\end{array}$ & 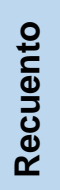 & $\begin{array}{c}\% \text { del } \\
\mathbf{N} \text { de } \\
\text { fila }\end{array}$ & 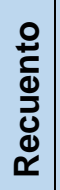 & $\begin{array}{c}\% \text { del } \\
\text { N de } \\
\text { fila }\end{array}$ & 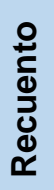 & $\begin{array}{c}\% \text { del } \\
\text { N de } \\
\text { fila }\end{array}$ & 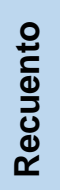 & $\begin{array}{c}\% \text { del } \\
\mathbf{N} \text { de } \\
\text { fila }\end{array}$ \\
\hline $\begin{array}{l}\text { 10. En } \\
\text { su } \\
\text { Hogar, } \\
\text { ¿tiene } \\
\text { Juegos } \\
\text { Electróni } \\
\text { cos? }\end{array}$ & Total & 11 & $10,1 \%$ & 72 & $66,1 \%$ & 21 & $19,3 \%$ & 4 & $3,7 \%$ & 1 &, $9 \%$ & $\begin{array}{c}10 \\
9 \\
10 \\
9\end{array}$ & $100,0 \%$ \\
\hline
\end{tabular}

Fuente: Arízaga y Arteaga (2018)

El análisis de la tabla se realiza en base a los juegos electrónicos que tiene el niño en su hogar y la relación con las horas que dedica el niño/a al entretenimiento electrónico. Como

\footnotetext{
“Visión de Futuro" Año 16, Volumen N²3 N¹, Enero - Junio 2019 - Pág. 157 - 176

URL de la Revista: http://revistacientifica.fce.unam.edu.ar/

URL del Documento: http://revistacientifica.fce.unam.edu.ar/index.php?option=com_content\&view=article\&id=492\&ltemid=101

ISSN 1668 - 8708 - Versión en Línea

E-mail: revistacientifica@fce.unam.edu.ar
} 
se observa en el cuadro, el $66,1 \%$ de los niños suelen pasar hasta 2 horas en este tipo de entretenimiento, mientras que en segundo lugar, con 19,3\%, se encuentran los niños que pasan de 2 a 5 horas en el mismo tipo de entretenimiento electrónico. Es importante conocer este tipo de análisis ya que, basándose de las entrevistas a los expertos, comentan que los niños actualmente pasan más tiempo en casa jugando con dispositivos electrónicos que en otros lugares que podrían ser más recreativos y saludables.

Se conoce que las horas que un niño puede pasar dentro de casa jugando con dispositivos electrónicos puede ser una de las principales causas para que desarrolle obesidad, ya que el niño/a deja de hacer todo tipo de actividad física sin conocer cuáles son los posibles problemas en la salud. Este estudio está estrechamente relacionado con la tecnología de hoy en día y cómo la publicidad incurre en la mente de los niños.

\section{Impacto de la publicidad en los niños/as con respecto a algún grado de sobrepeso y obesidad detectado por el médico}

Tabla N'14. Impacto de la publicidad en los niños/a s con respecto a algún grado de sobrepeso y obesidad detectado por el médico

\begin{tabular}{|c|c|c|c|c|c|c|c|c|c|}
\hline & \multicolumn{8}{|c|}{$\begin{array}{l}\text { obesidad detectado por el medico } \\
\text { MAYOR IMPACTO DE LA PUBLICIDAD PARA EL NIÑO/A }\end{array}$} \\
\hline & & \multicolumn{2}{|c|}{ Transporte Público } & \multicolumn{2}{|c|}{ Vallas Publicitarias } & \multicolumn{2}{|c|}{ Carritos de Comida } & \multicolumn{2}{|c|}{ Total } \\
\hline & & Recuento & $\begin{array}{c}\% \text { del N } \\
\text { de } \\
\text { columna }\end{array}$ & Recuento & $\begin{array}{c}\text { \% del } \mathrm{N} \\
\text { de } \\
\text { columna }\end{array}$ & Recuento & $\begin{array}{c}\% \text { del N } \\
\text { de } \\
\text { columna }\end{array}$ & Recuento & $\begin{array}{c}\text { \% del N } \\
\text { de } \\
\text { columna }\end{array}$ \\
\hline $\begin{array}{l}\text { 16. EN LA } \\
\text { ÚLTIMA }\end{array}$ & $\begin{array}{l}\text { Algún grado } \\
\text { de sobrepeso }\end{array}$ & 32 & $82,1 \%$ & 110 & $90,2 \%$ & 100 & $87,0 \%$ & 242 & $87,7 \%$ \\
\hline $\begin{array}{l}\text { VISITA AL } \\
\text { MÉDICO SU }\end{array}$ & $\begin{array}{l}\text { Algún grado } \\
\text { de obesidad }\end{array}$ & 7 & $17,9 \%$ & 12 & $9,8 \%$ & 15 & $13,0 \%$ & 34 & $12,3 \%$ \\
\hline PRESENTÓ: & Total & 39 & $100,0 \%$ & 122 & $100,0 \%$ & 115 & $100,0 \%$ & 276 & $100,0 \%$ \\
\hline
\end{tabular}

Fuente: Arízaga y Arteaga (2018)

La relación que se presenta en la tabla, sobre algún grado de obesidad y el impacto que tiene la publicidad en el niño, es de vital importancia. Muchos niños, son influenciados directamente por la publicidad que entra por sus ojos; cuando se observa constantemente algún tipo de valla publicitaria, este llega a posicionarse en su mente. Por esa razón, se necesita identificar si este tipo de publicidad llega a incidir en el desarrollo de enfermedades crónicas como el sobrepeso y la obesidad infantil, que reducen la vida de las personas.

Al identificar algún grado de sobrepeso, se determinar que puede estar dado principalmente por la publicidad que se encuentra en las vallas publicitarias con $90,2 \%$ de

\footnotetext{
“Visión de Futuro" Año 16, Volumen Nº 23 Nº1, Enero - Junio 2019 - Pág. 157 - 176

URL de la Revista: http://revistacientifica.fce.unam.edu.ar/

URL del Documento: http://revistacientifica.fce.unam.edu.ar/index.php?option=com_content\&view=article\&id=492\&ltemid=101 
incidencia en los niños, pero también en la misma categoría de vallas publicitarias puede llegar a afectar a un $9,8 \%$ de niños que pueden llegar a tener algún grado de obesidad infantil. Como podemos analizar, el grado de sobrepeso que puede tener un niño por la influencia de vallas publicitarias es un nivel mayoritario que el de obesidad.

\section{Invasión de la tecnología en los espacios verdes y las horas de entretenimiento electrónico del niño/a}

Tabla N15. Invasión de la tecnología en los espaci os verdes y las horas de entretenimiento electrónico del niño/a

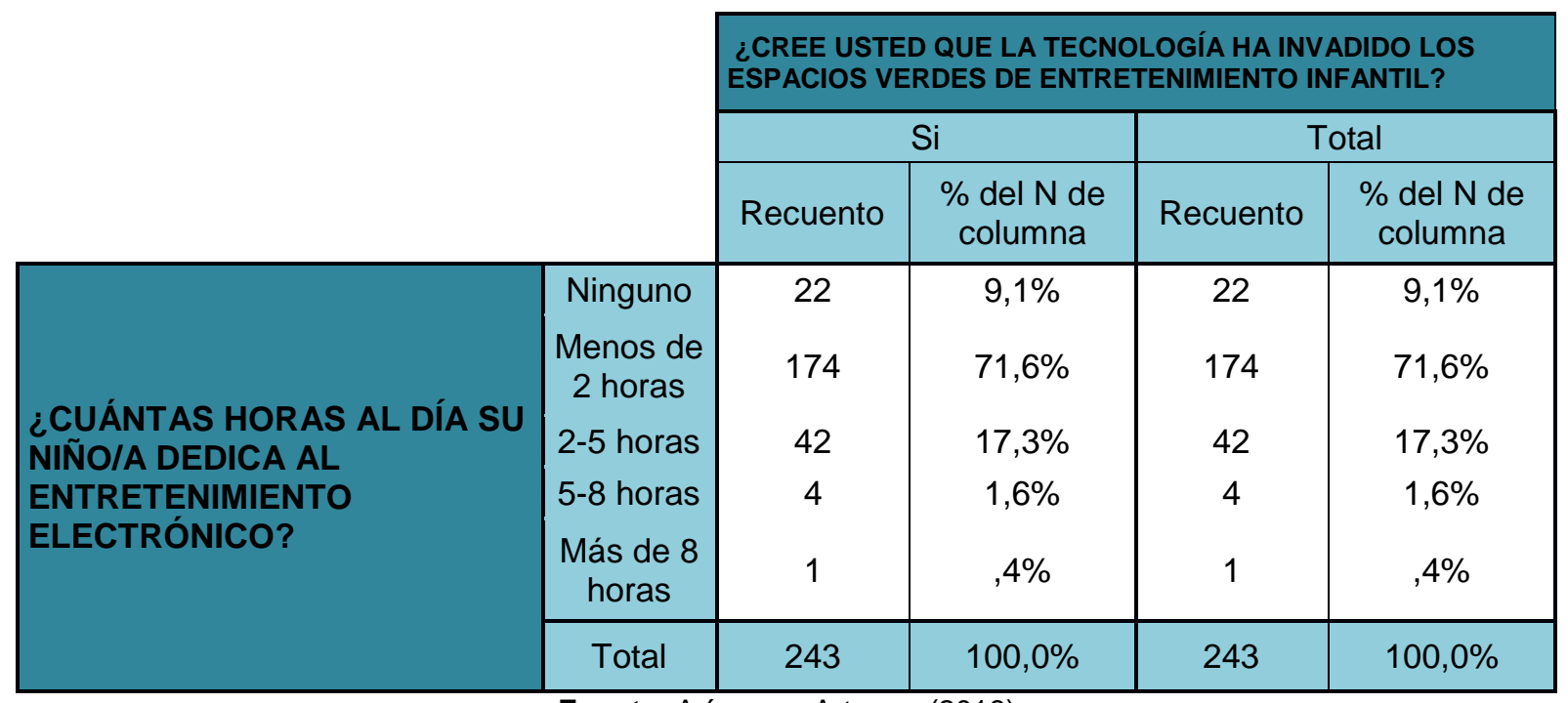

Fuente: Arízaga y Arteaga (2018)

Los resultados prueban que, de las personas encuestadas, el 71,6\% opinan que sus niños/as pasan hasta 2 horas en este tipo de entretenimiento tecnológico. Siguiendo a este primer grupo, tenemos el 17,3\% que representa a los niños/as que pasan desde 2 hasta 5 horas en el mismo tipo de entretenimiento tecnológico en lugar de otros espacios fuera de casa. Estos dos porcentajes expuestos son los que tienen mayor relevancia en la investigación y nos demuestra que realmente sí existe un reemplazo en los puntos verdes y en los estilos de vida de las personas. Estos porcentajes llegan a ser alarmantes, porque se puede apreciar el ausentismo de los padres diariamente ya que llegan a tener muy poco conocimiento de lo que los niños hacen en su tiempo libre.

Finalmente, después de haber analizado las variables, podemos determinar que se halla una estrecha relación en la invasión de la tecnología en los espacios verdes y las horas que los niños/as pasan entretenidos en la tecnología, se puede observar claramente este reemplazo y cambios de hábitos. Los expertos en niños recomiendan que estos tengan otras actividades fuera de casa y alejados de la tecnología, con alimentos sanos para una correcta nutrición. Como se ha mencionado en la entrevista a expertos, pediatras y nutricionistas “Visión de Futuro" Año 16, Volumen Nº 23 Nº1, Enero - Junio 2019 - Pág. 157 - 176 URL de la Revista: http://revistacientifica.fce.unam.edu.ar/

URL del Documento: http://revistacientifica.fce.unam.edu.ar/index.php?option=com_content\&view=article\&id=492\&ltemid=101 ISSN 1668 - 8708 - Versión en Línea

E-mail: revistacientifica@fce.unam.edu.ar 
entrevistados hablan con los padres de familia constantemente para que sus hijos tengan una mejor calidad de vida en el presente y sobre todo en un futuro.

\section{Los medios electrónicos que el niño/a está más expuesto a la publicidad con respecto} a las visitas al médico el niño/a presentó algún grado de sobrepeso

\begin{tabular}{|c|c|c|c|c|c|c|}
\hline & & \multicolumn{5}{|c|}{$\begin{array}{l}\text { ¿A QUÉ MEDIO ESTÁ MÁS EXPUESTO/A SU NIÑO/A A } \\
\text { LA PUBLICIDAD? }\end{array}$} \\
\hline & & $\begin{array}{l}\text { Redes } \\
\text { Sociales }\end{array}$ & $\begin{array}{l}\text { Juegos y } \\
\text { aplicaciones }\end{array}$ & $\begin{array}{l}\text { Páginas } \\
\text { Web }\end{array}$ & $\begin{array}{c}\text { Consolas } \\
\text { de Video } \\
\text { Juego }\end{array}$ & Total \\
\hline & & $\begin{array}{c}\% \text { del } \mathrm{N} \\
\text { de } \\
\text { columna }\end{array}$ & $\begin{array}{l}\% \text { del } \mathrm{N} \text { de } \\
\text { columna }\end{array}$ & $\begin{array}{c}\% \text { del } \mathrm{N} \\
\text { de } \\
\text { columna }\end{array}$ & $\begin{array}{l}\% \text { del } \mathrm{N} \text { de } \\
\text { columna }\end{array}$ & $\begin{array}{c}\% \text { del } N \\
\text { de } \\
\text { columna }\end{array}$ \\
\hline \multirow{3}{*}{$\begin{array}{c}\text { EN LAS VISITAS AL } \\
\text { MÉDICO SU NIÑO/A, } \\
\text { ¿PRESENTÓ ALGÚN } \\
\text { GRADO DE } \\
\text { SOBREPESO? }\end{array}$} & $\begin{array}{l}\text { Algún grado } \\
\text { de } \\
\text { sobrepeso }\end{array}$ & $89,7 \%$ & $90,5 \%$ & $87,2 \%$ & $50,0 \%$ & $86,5 \%$ \\
\hline & $\begin{array}{l}\text { Algún grado } \\
\text { de obesidad }\end{array}$ & $10,3 \%$ & $9,5 \%$ & $12,8 \%$ & $50,0 \%$ & $13,5 \%$ \\
\hline & Total & $100,0 \%$ & $100,0 \%$ & $100,0 \%$ & $100,0 \%$ & $100,0 \%$ \\
\hline
\end{tabular}

Fuente: Arízaga y Arteaga (2018)

En la tabla presente, es necesario analizar cuáles son los medios electrónicos a los que un niño/a esta más expuesto cada día y además, este se puede llegar a relacionar con algún grado de sobrepeso o de obesidad infantil. Según la entrevista a expertos, los niños actualmente se involucran o se exponen demasiado a la publicidad que se encuentra en redes sociales, juegos, aplicaciones, páginas web y consolas de video juegos, lo que puede llegar a ser uno de los factores principales que causa sobrepeso y obesidad en temprana edad.

De igual forma, podemos analizar esta tabla por categorías, identificando las más importantes para el estudio; en primer lugar, tenemos la categoría de juegos y aplicaciones, porque es la que presenta el porcentaje más alto con un $90,5 \%$ de representantes que han indicado que esta misma categoría presenta en los niños algún grado de sobrepeso y la diferencia que es del 9,5\% presenta algún grado de obesidad infantil. Es decir, que en su totalidad genera mucho sedentarismo.

\footnotetext{
“Visión de Futuro" Año 16, Volumen No 23 Nº1, Enero - Junio 2019 - Pág. 157 - 176

URL de la Revista: http://revistacientifica.fce.unam.edu.ar/

URL del Documento: http://revistacientifica.fce.unam.edu.ar/index.php?option=com_content\&view=article\&id=492\&ltemid=101

ISSN 1668 - 8708 - Versión en Línea

E-mail: revistacientifica@fce.unam.edu.ar
} 


\section{Impacto que tiene el niño en medios BTL con respecto a la edad del mismo}

Tabla N17. Impacto que tiene el niño en medios BTL con respecto a la edad del mismo

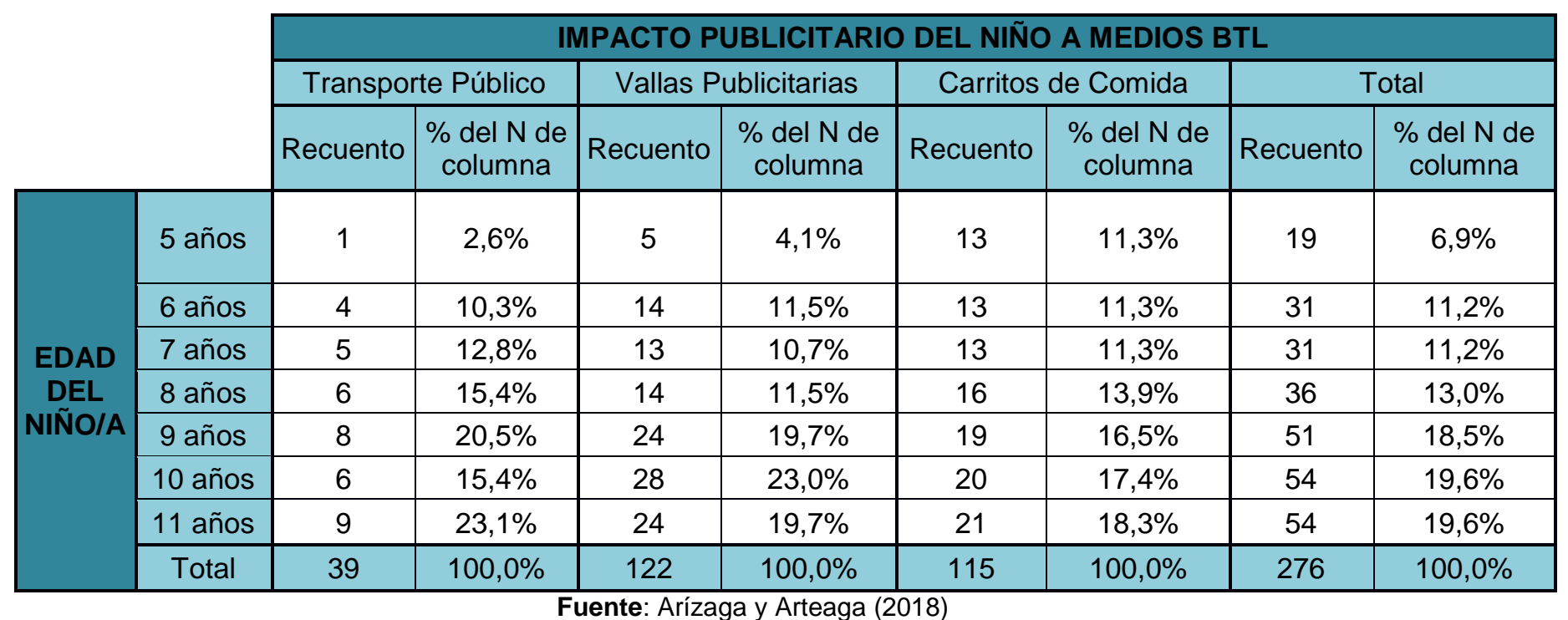

El impacto publicitario en distintos medios BTL como son transporte público, vallas publicitarias y carritos de comida pueden tener influencia de acuerdo a la edad entre 5 a 11 años, por lo que en la tabla se puede observar que el $23,1 \%$ de los niños/a de 11 años les llama la atención la publicidad que se expone en el transporte público como son buses, taxis, entre otros. Mientras que en los niños de 10 años de edad se enfocan en la publicidad de las vallas publicitarias donde se puede observar distintos tipos de campañas de alimentos no nutricionales, que al quedar grabados en la mente del niño pueden llegar a cambiar la decisión de compra de productos de una misma categoría.

Finalmente, los niños de 5 años están expuestos a la publicidad en carritos de comida como son carros de helados, hot dogs, hamburguesas, entre otros, por lo que incentivan a la decisión de compra inmediata de comida muy poco saludable para su organismo y que podría generar enfermedades como es la obesidad o sobrepeso infantil.

\section{CONCLUSIÓN}

Las encuestas se enviaron a 4.500 representantes entre escuelas fiscales, privadas y fiscomisionadas, pero regresaron 2.500 debido a que esta encuesta era de carácter opcional; únicamente 300 encuestas fueron efectivas para el proyecto, y de este número 216 fueron contestadas por representantes de escuelas privadas, 19 por públicas y 65 de escuelas fiscomisionadas, por lo que se puede observar la disposición y el interés que existe entre los

\footnotetext{
"Visión de Futuro" Año 16, Volumen No 23 Nº1, Enero - Junio 2019 - Pág. 157 - 176

URL de la Revista: http://revistacientifica.fce.unam.edu.ar/

URL del Documento: http://revistacientifica.fce.unam.edu.ar/index.php?option=com_content\&view=article\&id=492\&ltemid=101 
distintos grupos de referencia. De las 300 encuestas el $47 \%$ de las mismas tienen un nivel de instrucción de tercer nivel, por lo que con esta información recolectada se pueden obtener datos relevantes sobre las costumbres que las familias en la ciudad de Cuenca pueden tener.

Un logro para la investigación es que se puede analizar a niños/a de entre 5 a 11 años edad, pero con un número aproximado de niños y niñas que representan un porcentaje similar. Algo importante en esta investigación es el servicio de internet que tiene el hogar, ya que muchos de los medios electrónicos como son la Tablet, el celular, consola de video juegos y la computadora necesitan de estas herramientas para poder funcionar correctamente, pero sobre todo poder usarlos eficientemente, ya que existen un sinfín de aplicaciones que a los niños les gusta y gastan mucho de su tiempo en estas actividades.

Un factor fundamental que causa la obesidad infantil, es el hecho de que el niño/a no tenga la actividad física suficiente para su edad, por lo que gracias a la investigación de tablas de frecuencia podemos observar que el $57,35 \%$ de los infantes no realizan una actividad física fuera de su horas de clase, lo cual influye directamente a ganar peso y así llegar a tener obesidad infantil.

La accesibilidad de los niños a los medios electrónicos es inimaginable, por lo que gracias a la investigación se pudo observar que el medio más accesible para los niños es la computadora, porque allí encuentran juegos, páginas web, aplicaciones, entro otros y el niño está dispuesto a gastar su tiempo en este medio.

Al cruzar variables como son accesibilidad de medios electrónicos que tiene el niño y con quién pasa su tiempo libre, se obtuvieron resultados necesarios para la identificación del comportamiento que tienen padres e hijos dentro de la casa e inclusive se ha podido determinar cómo actúan también fuera de ella, por lo que muestra que muchas veces el niño tiene obesidad infantil o sobrepeso siendo cuidado por sus padres.

Se conoce que el $66,1 \%$ de los niños están profundamente inmersos en la tecnología por lo que muchas veces provoca que dediquen horas enteras jugando en internet y buscando distintos tipos de información, ya sea por entretenimiento o académicamente, por lo que es una causa principal que los niños hayan dejado de salir a jugar en áreas verdes y recreativas, y que esto influya en el sobrepeso que pueden llegar a tener.

Uno de los esfuerzos mayores que ha hecho el gobierno es la semaforización de los todos los productos comestibles, por lo que se cruzaron variables para ver que tanto influía esta nueva información en la decisión de compra de los representantes, y sus resultados fueron que el $78,8 \%$ de las personas eligen de mejor manera los alimentos saludables para los niños/as y toda la familia.

\footnotetext{
“Visión de Futuro" Año 16, Volumen Nº 23 Nº1, Enero - Junio 2019 - Pág. 157 - 176

URL de la Revista: http://revistacientifica.fce.unam.edu.ar/

URL del Documento: http://revistacientifica.fce.unam.edu.ar/index.php?option=com_content\&view=article\&id=492\&ltemid=101

ISSN 1668 - 8708 - Versión en Línea

E-mail: revistacientifica@fce.unam.edu.ar
} 
En conclusión, los medios electrónicos y la publicidad BTL influyen de gran manera en la decisión de compra de un niño/a y su representante, los dispositivos electrónicos tienen gran cabida en los niños en un rango de 11 a 5 años de edad por lo que son muy susceptibles a ese tipo de publicidad no convencional. Se genera mayor porcentaje de sobrepeso y no de obesidad infantil, por lo que este puede ser el factor para que en generaciones futuras dispare esta enfermedad, ya que lastimosamente es todo cuestión de tiempo.

\section{REFERENCIAS}

Aaker, D., \& Joachimsthaler, E. (2005). Liderazgo de marca. Bilbao, España, Ediciones Deusto.

Alonso Vázquez, M. (2006). Marketing Social Corporativo. Barcelona: eumed.net.

American Marekting Association. (2013, Julio). Recuperado de https://www.ama.org/AboutAMA/Pages/Definition-of-Marketing.aspx

Andreasen, A. (1995). Marketing Relacional.

Bonello, M. (2009, Diciembre 16). Eye to eye. Innovando en contacto real con marcas.

Ceberio, I. (2016). Patia. Recuperado de http://www.patiadiabetes.com/mx/frenar-obesidadinfantil/

Centro de Recursos de Promoción y EpS. (2017, Junio 16). Murcia Salud. Recuperado de http://blogs.murciasalud.es/edusalud/2017/06/16/influencia-de-la-publicidad-en-laalimentacion-infantil/

Deep, D. D. (2014, Noviembre 21). in Slideshare. Recuperado de https://es.slideshare.net/diegodanieldeep/atl-41862501

Forero Santos, J. (2010). El Marketing Social como Estratégia para la Promoción de la Salud. Freire, W., \& Ramírez, M. (n.d.). Encuesta Nacional de Salud y Nutrición. Recuperado de http://www.salud.gob.ec/encuesta-nacional-de-salud-y-nutricion-ensanut/

Harrison Garrido, J. (2010, Junio 18). El BTL, una alternativa y publicitaria.

Hernández Sampieri, R., \& Fernández Collado, C. (2006). In Metodología de la Investigación (p. 241). México D.F: McGraw-Hill Interamericana.

Keller, K. (2008). Administración Estratégica de Marca. Naucalpan de Juárez, México: Pearson Editorial.

Kotler, P., \& Armstrong, G. (2012). In Marketing (Décimocuarta ed., p. 720). México: Pearson Education Inc.

Kotler, P., \& Armstrong, G. (2017). Fundamentos del Marketing (13th ed.). México DF, México: Pearson Educación de México S.A.

\footnotetext{
“Visión de Futuro" Año 16, Volumen Nº 23 Nº1, Enero - Junio 2019 - Pág. 157 - 176

URL de la Revista: http://revistacientifica.fce.unam.edu.ar/

URL del Documento: http://revistacientifica.fce.unam.edu.ar/index.php?option=com_content\&view=article\&id=492\&ltemid=101

ISSN 1668 - 8708 - Versión en Línea

E-mail: revistacientifica@fce.unam.edu.ar
} 
Kotler, P., \& Roberto, L. (1992). Marketing Social. Ediciones Diaz de Santos, S.A.

Lindstrom, M. (2008). Buyology. Barcelona, España: Gestión 2000.

Mercado H, S. (2004). Mercadoténica Programada. México D.F: Grupo Noriega Editores.

Monge, S. (n.d.). neuromarca. Recuperado de http://neuromarca.com/neuromarketing/

Mosquera, R. (2014). hcjb más. Recuperado de http://radiohcjb.org/obesidad-infantil-en-elecuador/

Organización Mundial de la Salud. (2016, Junio). Organización Mundial de la Salud. Recuperado de http:/www.who.int/mediacentre/factsheets/fs311/es/

Pérez del Campo, E. (2002). La comunicación fuera de los medios (Below The Line). Madrid, España, ESIC Editorial.

Pérez Romero, L. (2004). Marketing Social. Pearson Educación.

Ramírez Reyes, C. (2009, Julio). Modelo Estratégico para BTL.

Real Academía Española de la Lengua. (2017). Real Academia Española. Recuperado de http://dle.rae.es/?id=apfpA3Q

Rodríguez Rossi, R. (n.d.). La obesidad infantil y los efectos de los medios electrónicos de comunicación. Recuperado de http://www.medigraphic.com/pdfs/invsal/isg2006/isg062g.pdf

Salud, Organización Mundial de la Salud. (2017). Organización Mundial de la Salud. Recuperado de http://www.who.int/mediacentre/news/releases/2017/increasechildhood-obesity/es/

Thompson, I. (2005, Julio). PromonegocioS.net. Recuperado de https://www.promonegocios.net/mercadotecnia/publicidad-historia.html

UNICEF. (2014). UNICEF. Recuperado de https://www.unicef.org/ecuador/media_27842.htm Unión Europea. (2011). Libro Verde de la Comisión Europea.

Zaltman, G., \& Kotler, P. (1971). El Marketing Social: Un acercamiento hacia la planeación de los cambios sociales. Journal of Marketing

\section{RESUMEN BIOGRÁFICO}

\section{Marco Antonio Ríos Ponce}

$\mathrm{PhD}$ (c) en Administración; Máster en Administración de Empresas; Postgrado en Marketing en la Industria Farmacéutica; Especialista en Administración de Empresas; Ingeniero Comercial; DocenteInvestigador Universidad del Azuay; Coordinador de la Carrera de Marketing; Editor General Revista UDAAKADEM. E-mail: marcoantonioriosponce@gmail.com

\footnotetext{
“Visión de Futuro" Año 16, Volumen No 23 Nº1, Enero - Junio 2019 - Pág. 157 - 176

URL de la Revista: http://revistacientifica.fce.unam.edu.ar/

URL del Documento: http://revistacientifica.fce.unam.edu.ar/index.php?option=com_content\&view=article\&id=492\&ltemid=101

ISSN 1668 - 8708 - Versión en Línea

E-mail: revistacientifica@fce.unam.edu.ar
} 


\section{Juan Francisco Álvarez Valencia}

Magister en Administración de Empresas mención Marketing, Universidad de Belgrano, Buenos Aires, Ingeniero Comercial, Docente-Investigador, Coordinador de Proyectos de Vinculación con la Sociedad, Universidad del Azuay. E-mail: falvarezv@uazuay.edu.ec

\section{Joffre Sebastián Arteaga Huiracocha}

Ingeniero en Marketing - Universidad del Azuay - Ecuador. E-mail: itoartega55@gmail.com

\section{Diana Carolina Arízaga Toledo}

Ingeniero en Marketing - Universidad del Azuay - Ecuador. E-mail: dianacaroarizagat@yahoo.com 